\title{
Metodología en la intervención de las pinturas de Qusayr 'Amra, Jordania
}

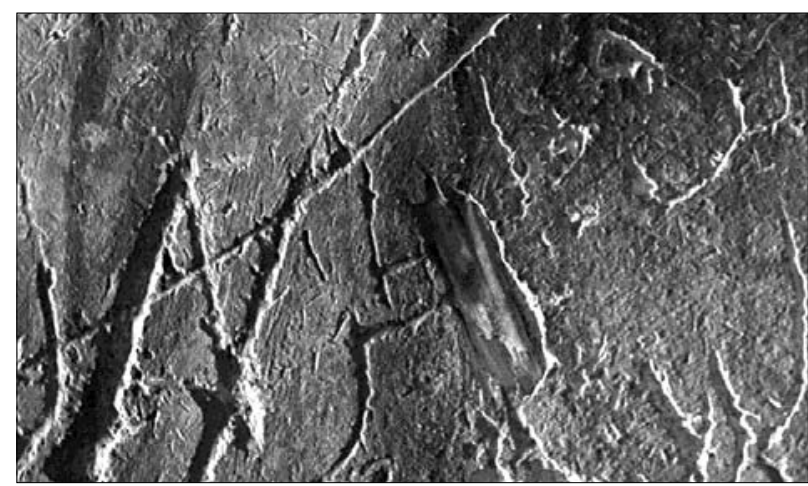

Víctor Jesús Medina Flórez Ana García Bueno

Departamento de Pintura

Universidad de Granada

\section{Resumen}

Este trabajo se integra en el proyecto de restauración del monumento dirigido por el arquitecto Dr. Almagro Gorbea, de la Escuela de Estudios Árabes (CSIC) financiado por el Ministerio de Asuntos Exteriores. Recoge los resultados del trabajo realizado desde el 15 de octubre al I 5 de diciembre del 96, con la colaboración de un equipo de restauradores, para estudiar los materiales y la técnica de las pinturas murales de Qusayr 'Amra, Jordania, así como para definir una metodología apropiada para acometer la restauración de dichas pinturas con objeto de valorar y planificar el desarrollo de la intervención que se debería llevar a cabo en un futuro. A pesar de los resultados obtenidos la intervención ésta no se ha desarrollado todavía por problemas de financiación.

El Departamento de Pintura de la Facultad de Bellas Artes de Granada viene trabajando durante los últimos años en una línea de investigación relacionada con el conocimiento de los materiales empleados así como de la técnica y procesos de ejecución de la pintura mural hispanomusulmana y su conservaciónrestauración. Las pinturas de Qusayr 'Amra (siglo VIII) constituyen uno de los ejemplos más interesantes, no sólo de la pintura del periodo Omeya, sino de todo el arte Islámico'. Su estudio resulta de sumo interés dentro de esta línea de trabajo ya que aporta datos que consideramos fundamentales a la hora de establecer las raíces de la técnica de pintura mural hispanomusulmana.

\section{Palabras Clave}

Restauración / Pintura mural / Qusayr 'Amra / Pintura musulmana / Técnicas pictóricas

\section{INTRODUCCIÓN: LA RESIDENCIA CALIFAL DE QUSAYR 'AMRA}

Qusayr 'Amra está a unos $100 \mathrm{Km}$. al E. de Amman, en plena zona desértica, en medio del amplio lecho seco del Wadi Butum, que algunos años corre hasta la depresión de Azraq. El cauce del Wadi Butum origina una extensa región idónea para la caza en el desierto, tema que estará muy presente en la representación iconográfica de las pinturas.

Posiblemente fue construida en el reinado del califa Walid I, entre el 7II y el 7|5. Dicha cronología se fundamenta en la identificación de una inscripción de las pinturas del monumento, en la cual consta el nombre de los monarcas vencidos por el islam durante el reinado del califa Omeya Walid I (705-7I5). Puesto que éste murió en el 715 y Rodrigo, último rey de la monarquía visigoda y uno de los reyes representados, fue vencido en Guadalete en el 7II, las pinturas debieron realizarse pues en el periodo comprendido entre ambas fechas².

Qusayr 'Amra se sitúa cerca de otras residencias omeyas en un enclave próximo a las ricas comarcas de Siria y Palestina, territorios recién conquistados y sometidos 
al gobierno del califato y a su vez lejos de la Meca y Medina, ciudades santas del islam pero que siempre fueron fuente de problemas para la dinastía Omeya.

Estos palacios dispersos por el desierto no eran residencias permanentes, sino que servían de cobijo a la corte nómada de los primeros años del califato. En ellos los monarcas podían disfrutar de una vida similar a la del desierto arábigo del que procedían, controlando de cerca a las diferentes tribus de beduinos que eran su principal apoyo.

Qusayr 'Amra es una adaptación de unos baños romanos, en los que la sala fría se convierte en salón de recepciones de la corte 3 . Se puede decir que 'Amra, que pertenece a un periodo inicial del arte musulmán, tiene una clara influencia del arte Bizantino, ya que antes de la expansión del islam no había una tradición arquitectónica propia.

La estructura arquitectónica del monumento es muy interesante, consta de una la sala principal cubierta por tres bóvedas de cañón que se soportan en los muros interiores con arcos ligeramente apuntados de modo que, aun aparentando ser exteriormente tres estancias diferenciadas, el interior es un espacio bastante unitario. Al fondo, en una situación prioritaria, se encuentra el salón del trono, del que se accede a dos alcobas laterales.

A los baños se accede por la puerta que se abre en la nave izquierda de la sala principal. Son tres estancias distribuidas en $L$, la primera un apoditerium y dos caldarii. Estas últimas tenían un falso suelo bajo el cual circulaba el calor del horno que se situaba en el exterior, junto a la cisterna a la cual llegaba el agua desde el sistema hidráulico (el pozo y la noria).

En cuanto a la iconografía, también muestra una clara influencia del arte Bizantino. En Amra la temática es figurativa y ello se debe a que en un primer momento el arte musulmán no había hecho extensiva la prohibición de representar seres vivos a la arquitectura civil, dejando esta limitación solo para el arte religioso.

Los temas tratados se adaptan a las diferentes funciones de las estancias, la sala principal distribuye sus temas entre temas de caza, cacerías de onagros, y escenas de corte. Las escenas de caza tienen paralelismo con las pinturas de Dura-Europos y con un fresco de Susa, fechado en la primera mitad del siglo IV4. En la estancia derecha de esta sala principal se representan escenas de la corte, los reyes representados a la izquierda de la figura femenina principal, se identifican como los monarcas vencidos por el Islam, anteriormente aludidos ${ }^{5}$.

En el salón del trono se representa en su frontal al califa guardado por dos personajes, en los paramentos laterales de esta estancia se representan personajes de la corte y figuras femeninas que deben tener relación con alegorías de la fecundidad y la abundancia.
A ambos lados de la sala del trono, como se ha dicho con anterioridad, a través de sendas puertas de pequeña altura, se accede a las habitaciones laterales, cuya función sería la de alcobas. En este caso la decoración pictórica, formada por un motivo de roleos con hojas de vid y racimos, arranca desde el suelo, a diferencia de las otras salas del edificio.

La bóveda de la primera estancia del baño está dividida en rombos por ramas de laurel que encuadran, fundamentalmente, figuras de animales. En la clave de la bóveda destacan tres bustos que representan las Tres edades de la vida. En el tímpano y los lunetos a ambos lados de la ventana se observan figuras humanas.

En el primer caldarium, denominado de las mujeres, la bóveda de crucería aparece decorada con motivos vegetales. En los tímpanos se pueden observar escenas íntimas de mujeres y niños en clara referencia al uso de esta estancia.

Finalmente, el caldarium del Califa, cubierto por una bóveda de media naranja que se sostiene sobre cuatro arcos, ofrece una representación del universo y los símbolos del Zodiaco.

\section{CONSIDERACIONES PREVIAS SOBRE LA TÉCNICA PICTÓRICA}

Como hemos visto, Qusayr 'Amra recoge una clara influencia del arte Bizantino, imperio al que se habían conquistado los territorios con los que se amplia el califato. En cuanto a la iconografía su relación con el arte helenístico es innegable, de él toma temas como la representación del califa, exacta a la que el arte bizantino hace del emperador en su divina maiestas, siguiendo la etiqueta de la corte que prescribía la inmovilidad y la frontalidad' . Todo esto también hace pensar que la influencia bizantina exista también a nivel técnico.

Del mundo bizantino nos queda constancia de los métodos empleados en la pintura mural a través de tratados como el de Dionisio de Fourna o el de Nektar y aunque son posteriores cronológicamente a las pinturas de Qusayr 'Amra, Mora, P. Mora, L. y Philippot, P. opinan que el método utilizado en periodos anteriores varía sustancialmente del descrito en estos tratados. Nektar dice que "en primer lugar hay que diluir la cal en una cubeta, para apagarla, y añadirle paja, lavada y bien cortada, de medio dedo de longitud, así como algo de arena. A continuación hay que agitarlo todo y dejarlo asentar durante tres días, hasta que la paja se vuelva blanda y se combine con la cal y la arena". La técnica descrita por él es claramente un fresco ya que dice textualmente "teniendo cuidado de pintar en un enlucido húmedo la obra será duradera"7. 


\section{OBJETIVOS Y METODOLOGÍA DE LA INTERVENCIÓN}

En la intervención desarrollada durante los días 15 de octubre al 15 de diciembre de 1996 se partía de la información obtenida tras un primer acercamiento in situ a la problemática general de las pinturas murales del monumento durante una visita previa de inspección efectuada en abril de 1996. En aquella ocasión se había realizado:

$1^{\circ}$.- Un examen organoléptico global, el cual consistió en una mera revisión visual del estado de conservación que presentaba toda la superficie pintada en los paramentos interiores, así como de las posibles causas de alteración que, tanto en el presente como en el pasado, pudieran haber incidido en el mismo.

$2^{\circ}$.- Un breve estudio analítico con objeto de completar los datos obtenidos en esta primera observación directa de la obra. Para ello se realizó una primera toma de muestras puntuales con objeto de someterlas a examen en laboratorio e identificar así algunos aspectos iniciales de interés que permitieran orientar la actuación que se pretendía realizar.

Aquel primer estudio, que no pretendía ser exhaustivo, fue suficiente para cumplir con los objetivos previstos y por ello, aunque sus resultados no se podían considerar como concluyentes y definitivos, fueron de gran utilidad para preparar la propuesta de intervención que debería ser puesta a punto durante esta primera campaña de actuación.

En aquel trabajo previo se apuntaron pues los primeros datos sobre algunos de los materiales y técnica empleados en la ejecución de las pinturas. También se aportaron otros que nos aproximan a un conocimiento de las posibles patologías a tratar y cuyo origen se vincula tanto a su propia ejecución como a la utilización de algunos tratamientos realizados en intervenciones anteriores ${ }^{8}$ cuya acción sobre la pintura podría haber sido la causa de algunas de las alteraciones que ésta presenta en la actualidad.

Esta primera valoración sobre el estado de conservación de las pinturas murales de Qusayr 'Amra sirvió para evidenciar la necesidad de una intervención de restauración en las mismas, en la cual se deberían abordar los siguientes objetivos:

Por una parte aspectos no resueltos o inacabados en la restauración anterior (tales como la limpieza, consolidación de morteros, la reintegración matérica y cromática de lagunas, etc.

Por otra parte otros que pudieran haberse producido con posterioridad (eliminación de sales, acumulación de suciedad superficial...) que también inciden en la conservación de las pinturas y que es preciso resolver y controlar.

Otras situaciones que se han desencadenado a raíz de los tratamientos realizados en la restauración de los años 70 que, además de repercutir en el aspecto estético de la obra están generando procesos de deterioro (tales como la aplicación del fijativo de protección y consolidantes...).

En algunos casos el origen de esas patologías, que requieren una intervención de forma más urgente, se encuentra vinculado de un modo más o menos directo a algunos problemas arquitectónicos que deberían ser resueltos previamente.

Dada la complejidad de los trabajos a realizar, las dimensiones de la obra y las dificultades de acceso derivadas de su ubicación (en el desierto de Jordania a $40 \mathrm{Km}$ de la población más cercana) se hacía necesario una planificación conjunta de la actuación del equipo responsable de la intervención arquitectónica del edificio y el de la restauración de las pinturas, así como también ordenar las intervenciones y su desarrollo temporal.

Así pues, en la campaña, puesto que aun no se había previsto iniciar la intervención arquitectónica, lo que se pretendía era realizar una experimentación de las propuestas de tratamientos previstos en una zona concreta, para poder valorar la eficacia de los mismos así como evaluar posteriormente costes y resultados.

Para concretar la propuesta de intervención en las pinturas se hacía necesario desarrollar un estudio más detallado. La información de la primera visita de inspección resultó de gran utilidad, tanto para orientar las actuaciones que se llevarían a cabo, como para hacer una primera sugerencia de tratamientos en base a la cual se realizó la selección de los materiales necesarios y su provisión, ya que era necesario enviar la mayoría de ellos desde España.

Con objeto de poner a punto los tratamientos se tomó una zona representativa de la problemática de la obra. La zona seleccionada fue el salón del trono ya que presentaba todos los problemas de alteración antes vistos. También se hicieron en otras zonas puntuales, pruebas de los mismos tratamientos para comprobar su efectividad. Para ello se hacía necesario completar los resultados del estudio material. Además los datos que nos aportaría una obra clave como esta resultarían de sumo interés para el conocimiento de las técnicas de la pintura mural musulmana.

El trabajo se planteó en la zona correspondiente al salón del trono, ampliando y contrastando la información obtenida como resultado del estudio realizado a partir de la primera visita de inspección. Así pues se completó la toma de muestras atendiendo aquellos aspectos que fueron suscitándose durante el trabajo en la zona seleccionada para poder realizar su posterior estudio una vez regresáramos a España.

Asimismo, dado el interés de los resultados, en futuras intervenciones, se deberían seguir haciendo igualmente estudios más completos de cada una de las zonas de la obra, tanto a nivel de morteros, aglutinantes y 
pigmentos como de patologías y alteraciones, ya que, según hemos podido observar no son absolutamente coincidentes en todo el conjunto monumental.

\section{Estudio material de las Pinturas del Salón del Trono}

\section{a. Morteros}

En Qusayr 'Amra el número y espesor de las capas de mortero varía según las zonas del muro, en algunos casos hay una sola capa que mide escasos milímetros y en otros encontramos dos niveles en los cuales las capas son más gruesas. Los espesores son muy diferentes ya que la fábrica es muy irregular. El primer mortero se aplica para nivelar pero en ocasiones la irregularidades son tan grandes que se dejan pequeñas zonas con la piedra a la vista sobre la que se aplica directamente el segundo mortero.

Como se pudo observar en varios puntos del monumento, por las deficientes condiciones de conservación en que se encontraban las pinturas, el sistema de agarre de los morteros es muy interesante. Se observaron dos procedimientos en el mortero de base para mejorar la adherencia del mortero que se superpone: uno formado por pequeños grupos de guijarros y otro a base de incisiones en espiga, ambos métodos empleados también en la técnica romana?.

Los resultados de este estudio nos permiten concluir que en todos los casos el componente mayoritario de los morteros es el carbonato Cálcico. La presencia de este material se debe a su empleo como aglomerante (producido por la carbonatación del $\mathrm{Ca}(\mathrm{OH})_{2}$ que reacciona con el $\mathrm{CO}_{2}$ atmosférico) y también como árido (una calcita muy blanca y pura, probablemente polvo de mármol). También se identificó de forma minoritaria arena, compuesta por cuarzo y feldespatos esencialmente.

Se ha podido comprobar la presencia de paja, que le proporciona una textura muy característica advertida tanto visualmente como en el microscopio óptico y SEM, que sin duda fue añadida intencionadamente para mejorar su trabazón y como reserva de humedad durante el proceso de carbonatación, lo que recuerda evidentemente la técnica bizantina ${ }^{10}$.

Por otra parte las muestras analizadas por DRX han dado como resultado la presencia de halita (cloruro sódico) en el mortero, llegando en algunas muestras a una proporción del $5 \%$. Su presencia se ha visto confirmada en el SEM por la identificación de cloro en la mayoría de las muestras analizadas.

\section{b. Pigmentos}

Las capas de pintura son sumamente finas y además parcialmente solubles en agua, lo que constituye una dificultad añadida de cara a la intervención, sobre todo en el caso de la limpieza. Los colores aparentes de la película pictórica examinados son: blanco, rojo, ocre, naranja, azul, verde, gris y negro.

El estudio de los pigmentos empleados en la decoración pictórica del monumento se deberá seguir completando, ya que los resultados obtenidos ofrecen datos de gran interés pero se limitan a zonas muy concretas del palacio, principalmente del salón del trono.

A continuación se exponen las conclusiones del estudio de pigmentos que se ha realizado, tanto con Microscopia Óptica como con el Microscopio Electrónico de Barrido (SEM).

BLANCOS: En los casos analizados, los pigmentos blancos empleados han sido el carbonato de calcio y el albayalde (blanco de plomo).

En las capas pictóricas blancas de base parece ser que en geereal se empleó el Carbonato Cálcico. Aunque en el uso de éste como pigmento el grano es menor y la pureza de los materiales mayor que en el mortero, realmente no hay una separación clara entre capa pictórica y mortero. El color del mortero parece que se utilizó en algunos casos como fondo de la pintura.

Sin embargo el empleo de albayalde como pigmento blanco se detecta solo en una de las muestras analizadas, este pigmento es conocido desde época clásica apareciendo citado en los escritos de Plinio.

ROJOS, OCRES y ANARANJADOS: En las muestras analizadas de estos colores, encontramos gran variedad de resultados, como veremos a continuación. Los pigmentos utilizados son hematites, minio y bermellón. Además se ha detectado también la presencia de oropimente o rejalgar.

En algunas muestras, el hierro ha sido el único elemento que ha dado color a la capa, constituida por una tierra rica en oxihidróxidos de hierro, principalmente Hematites. En otras muestras encontramos junto a los hematites otros elementos como por ej. el As y S estos elementos se explican por la utilización de oropimente o rejalgar para obtener modificaciones en el color o la de $\mathrm{Pb}$ que es indicativa de la utilización de minio ya que no se detectaron granos blancos en la capa que hicieran pensar en la utilización de albayalde en la mezcla.

Por último, en la otra muestra tomada del fondo de uno de los arcos cuyo tono de rojo es muy intenso, se identificó únicamente Hg. Podemos deducir que el único pigmento utilizado en este caso es el cinabrio o bermellón.

Los ocres tanto si se encuentran aplicados en carnaciones, como si corresponden a representaciones de animales, están compuestos por una tierra que contiene oxihidróxidos de hierro.

AZULES: En el caso del salón del trono se ha identificado un único pigmento azul en todas las muestras estudiadas, con características y composición muy si- 
milares. Solamente se diferencian por la cantidad de pigmento blanco presente en cada muestra.

El pigmento azul empleado es el Lapislázuli. La presencia de $\mathrm{Na}$, S y Si, elementos característicos del mismo, indica que está constituido por un Silicato de Sodio y Azufre, composición químicas de este pigmento. Se descartó la posibilidad de que pudiera tratarse de un vidrio coloreado tanto por las características ópticas como por la imagen de Back-Scatterd del SEM que nos permite observar el brillo de los granos que componen la capa pictórica, mucho mayor en este caso del que correspondería a un vidrio.

VERDES: Pensamos que puede tratarse de una Tierra Verde, un pigmento muy empleado en pintura mural y que es citado por autores clásicos como Plinio. Las tierras verdes son consecuencia de la meteorización de silicatos de hierro y por tanto tienen una composición química compleja en la que siempre aparece este elemento, en algunas muestras pueden observarse al microscopio óptico pequeñísimos granos de hematites.

GRISES Y NEGROS: Las alteraciones que presentan de forma generalizada las pinturas en esta zona, derivadas de su exposición al humo y al calor, hace que no esté claro el uso de un pigmento negro en algunas muestras de este apartado, ya que a pesar de que el color aparente de la capa sea gris no es seguro que en origen lo fueran.

Los pigmentos identificados en estas capas han sido: negro vegetal, oropimente o rejalgar, minio y probablemente negro de hueso y blanco de plomo.

En una de las muestras, correspondiente a un detalle de un capitel, el pigmento negro estaba situado entre el color naranja de base y el blanco, lo que indica que sobre la base naranja se aplicó una línea negra que enmarca el arco sobre la que está la capa discontinua de color blanco, que corresponde a los brillos de la decoración; por tanto el pigmento negro en este caso puede considerarse claramente original. No se ha identificado ningún elemento característico en la capa negra y además el número de cuentas baja considerablemente, por lo que puede decirse que en este caso se trata de un negro vegetal.

\section{c. Aglutinantes y aditivos orgánicos}

También se analizaron la posible presencia de materia orgánica utilizada como aglutinantes de la pintura, como aditivo de los morteros" y también procedente de los productos empleados en restauraciones anteriores. Únicamente se identificó la presencia de goma arábiga en las capas más superficiales de los mismos ${ }^{12}$.

Los resultados nos indican que la presencia de altas cantidades de arabinosa, así como el resto de los monosacáridos, evidencian la existencia de goma arábiga en el mortero, que puede tener su origen en el uso de este aditivo o en su filtración a través de las capas de pintura, ya que también se identificó este mismo material en las capas de pintura de las muestras analizadas. En todos los casos se identificó goma de acacia tanto en la capa más superficial de mortero como en la capa pictórica.

Por otra parte del estudio de materiales orgánicos se deduce que los materiales empleados como consolidantes en la restauración anterior fueron acetato de polivinilo, y goma laca, como fijativo y capa de protección. La alteración de ésta y su densidad explican el brillo y alto grado de amarilleamiento que se ha producido en la capa de recubrimiento.

Después de la valoración de los todos los datos obtenidos en el estudio de materiales y la observación directa de la obra se podrían plantear unas primeras conclusiones de carácter general en relación con la técnica de ejecución las pinturas de Qusayr 'Amra, las cuales deberán ser ampliadas y completadas con una mayor cantidad de datos que podrían obtenerse en el desarrollo de las siguientes fases de la intervención prevista, ello permitiría completar el estudio de las pinturas del monumento tanto a nivel formal como de materiales.

Los pigmentos han sido aglutinados con goma arábiga para su aplicación sobre una base de morteros de cal y polvo de mármol con adición de paja, arena.

La película pictórica parece ser extremadamente delicada, dado su escaso espesor y la falta de resistencia del aglutinante empleado para su ejecución, si bien en algunas zonas parece estar reforzada por la acción de una carbonatación superficial del hidróxido cálcico "de reserva" del mortero, tal y como se observa en el análisis de las estratigrafías por medio del SEM.

La complejidad de la decoración y el tamaño de algunos paños de la decoración sin que se observen en ningún caso líneas de jornadas, hace difícil pensar que su ejecución se realizase al fresco, lo que podría indicar que se trata de una técnica al $\operatorname{seco}^{13}$ en la que el aglutinante es la goma arábiga'14.

No obstante, la presencia de carbonato cálcico en las capas de pintura podría haber sido incrementada de forma voluntaria por la realización de politiones, con la intención de que la fijación de la pintura se viera reforzada por la acción de la carbonatación de la cal del mortero fresco, lo cual explicaría la inexistencia de jornadas.

Este proceso se hacía después de aplicar las grandes manchas de color y consistía en el pulido de la superficie pintada para hacer que los restos de humedad e hidróxido cálcico presentes en el interior del mortero migraran a la superficie y de este modo mejorar fijación de los pigmentos por la carbonatación que se producía a nivel superficial. Posteriormente también aglutinando con goma arábiga los pigmentos se aplicarían los detalles más minuciosos.

En cualquier caso es difícil asegurar con absoluta fiabilidad cual de las dos hipótesis sobre el proceso de 
$\longleftarrow$
$\square$ trabajo seguido es la cierta ya que el estudio sobre la composición material no nos aporta datos suficientes debido a que en ambos casos sería la misma.

Respecto a los pigmentos tenemos que volver a insistir en la riqueza cromática que debieron tener estas pinturas, dada la gran variedad de pigmentos identificados y la pureza de los mismos, albayalde, calcita, lapislázuli, cinabrio o bermellón, minio, oropimente o rejalgar, tierra roja y tierra verde.

En cuanto al dibujo no se han detectado líneas de dibujo inciso durante la intervención, sin embargo sí se han observado en algunas estratigrafías capas internas de color negro que podrían explicarse como lí-

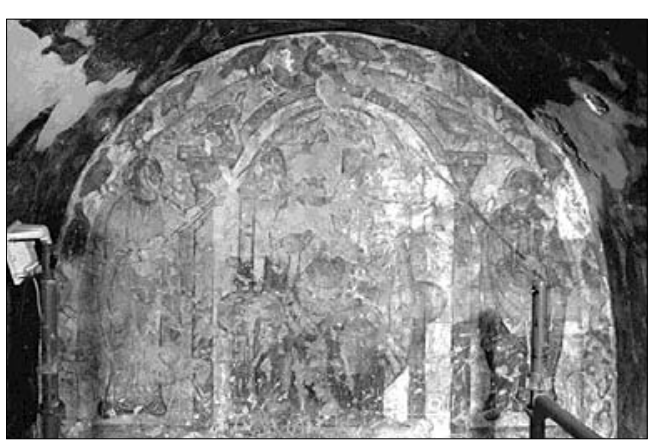

Fotografía 1

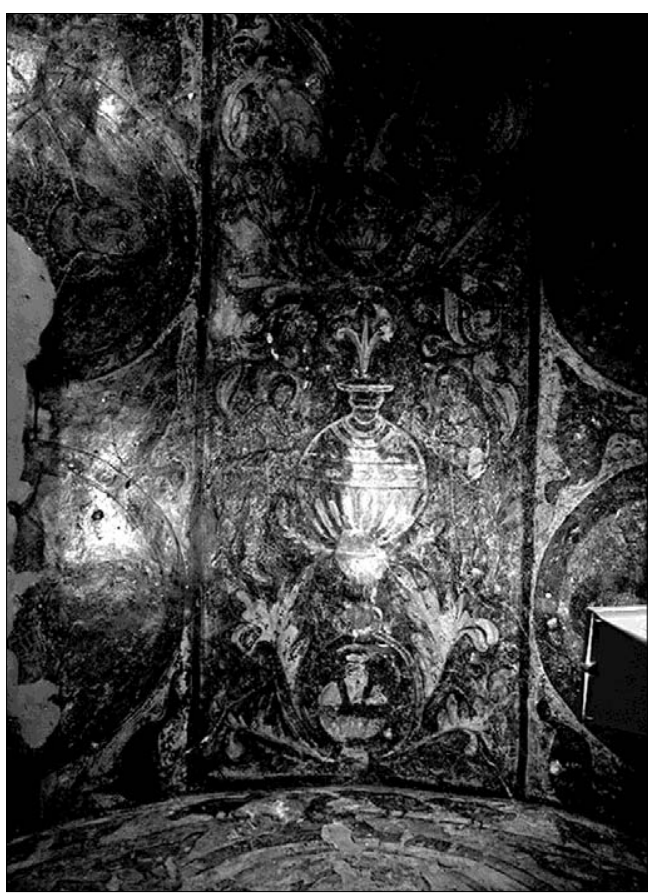

Fotografía 2

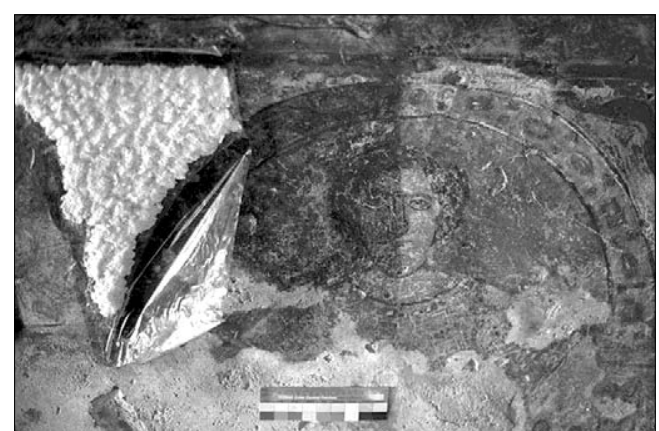

neas de un posible dibujo subyacente aunque no tenemos datos concluyentes en este sentido.

\section{Estado de conservación}

Como primera impresión general, resaltaba a simple vista el alto grado de amarilleamiento y brillo que presentan las pinturas, más acusado incluso aquí que en otras zonas del monumento (Fotografías I, 2), atribuibles a la presencia de fijativos de protección aplicados en restauraciones anteriores y a su alteración cromática por el envejecimiento sufrido desde entonces, ya que éstos se manifiestan incluso en las faltas antiguas del estrato pictórico (grafittis, golpes...) presentando este mismo aspecto brillante y amarillento a diferencia de otras más recientes en las que se observa el blanco intenso del mortero. Los resultados relativos a la naturaleza del consolidante utilizado en la sala del trono, obtenidos en el estudio de las muestras tomadas durante la primera visita de inspección, indicaban la presencia de goma laca y PVA en su composición.

Este fijativo estaba provocando además una gran tensión en la superficie del estrato pictórico dada la microfracturación que presentaba este mediante la observación puntual en algunas zonas con lupa de 10 aumentos.

Sobre el estrato de fijación se observa un abundante depósito de polvo superficial.

Por último, destaca el intenso ennegrecimiento de las pinturas en las zonas superiores, correspondiente al acúmulo de humo y suciedad más antigua endurecidos por procesos de recristalización de carbonatos y que no fue eliminado en los procesos de limpieza de las restauraciones anteriores (Fotografías 2, 4.1 y 5.1).

A través de una observación detallada se podía confirmar que la pintura estaba muy retocada. Algunos repintes se apreciaban, de forma evidente, reforzando perfiles o contornos en negro cuando los originales eran rojizos, o incluso recreando detalles de la decoración. Otros se superponían de una forma arbitraria en algunas pérdidas del color, y en las zonas en las que no había sido posible eliminar el ennegrecimiento del estrato pictórico ocultándolo o matizándolo.

Resalta la innumerable cantidad de grafittis incisos, algunos de fechas muy recientes, que se extendían por toda la zona inferior de las pinturas, hasta donde

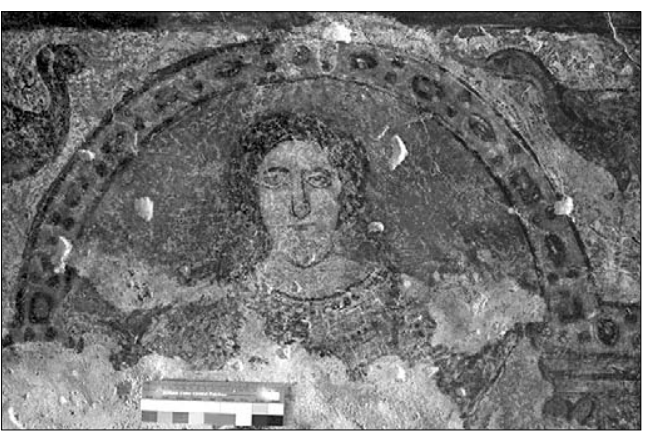




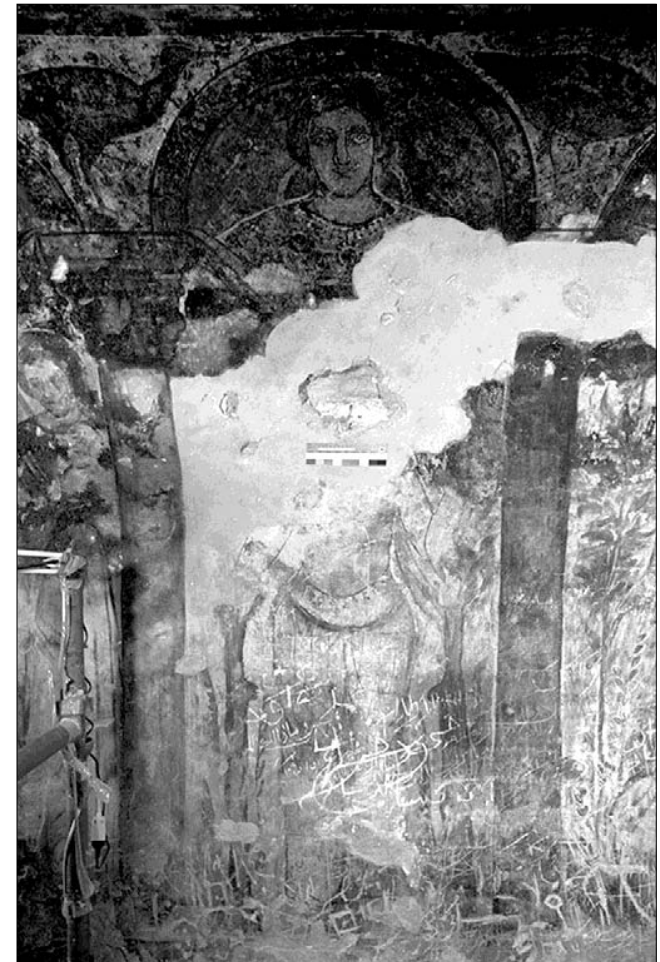

Fotografía 3

es posible el acceso, y que habían hecho desaparecer de forma lamentable una gran parte de la superficie pictórica (Fotografía 3).

De una forma puntual se ha podido detectar en varios lugares la presencia de cortes rectilíneos que delimitan zonas en las que se observan abundantes faltas, lo que parece ser un indicativo evidente de diversos intentos fallidos de arranques de fragmentos de las pinturas ${ }^{15}$.

Se observan también numerosas faltas de un tamaño considerable que afectan a los diferentes estratos de mortero y que presentan diferentes aspectos según las zonas, sin adivinarse en las mismas una uniformidad en el criterio de reintegración aplicado en la restauración anterior (Fotografía I). En algunos casos aparecen rellenas de mortero aplicado de un modo más o menos regular. En otras, los bordes de las faltas aparecen cogidos con algodón, impregnado en un adhesivo (PVA), en otras ocasiones éste rellena también toda la superficie de la laguna. Finalmente existen otras en las que no se aprecia relleno aparente.

Hay que destacar la importancia de las pérdidas de la película pictórica producidas por filtraciones de las cubiertas que han dado lugar a faltas muy importantes por su gran extensión. El mortero se presenta muy degradado en estas zonas y en algunas ocasiones las pérdidas afectan incluso a la fábrica. También se pudo comprobar la existencia de numerosas oquedades, algunas de ellas presentan deformaciones del estrato superficial por abolsamiento del mismo.

Los diferentes tipos de laguna, según el tratamiento matérico recibido para su relleno, se ven por el con-
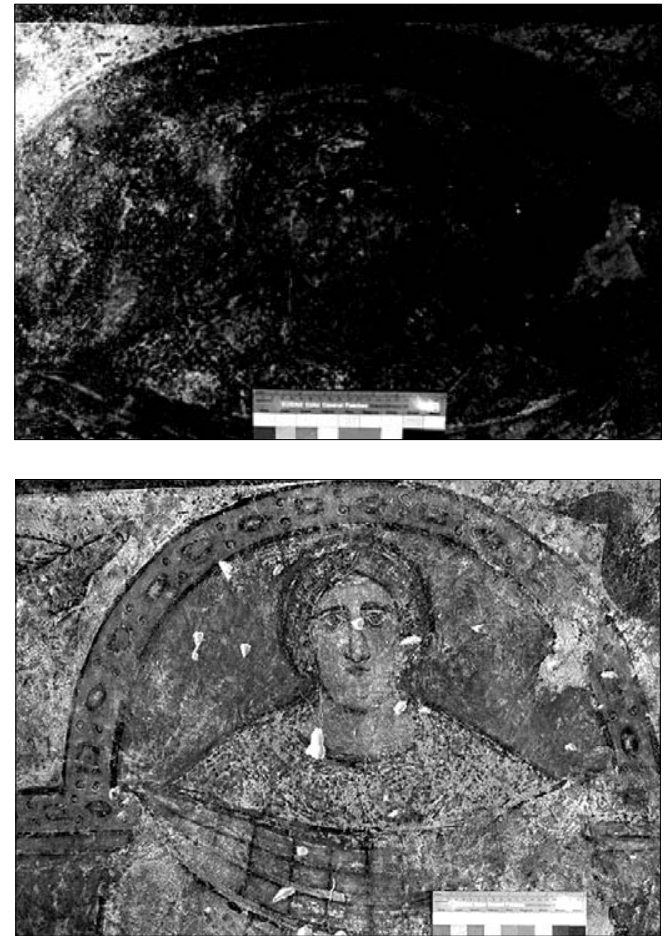

Fotografía 5.।

trario igualmente entonados cromáticamente como resultado de alguna intervención realizada con anterioridad. Esta reintegración había sido realizada por medio de una tinta plana, que en la mayoría de los casos no resulta tan neutra como sería deseable.

\section{INTERVENCIÓN}

El trabajo se desarrolló durante un total de 2.500 horas (realizadas por un equipo de seis restauradores de la Facultad de Bellas Artes de Granada). Se comenzó con la observación pormenorizada y el correspondiente mapeo exhaustivo de las alteraciones de la zona seleccionada para la intervención. Posteriormente se realizaron pruebas de tratamientos en base a los resultados obtenidos en el estudio preliminar. Finalmente se aplicaron éstos en la zona seleccionada para realizar una valoración temporal y de este modo, extrapolando los resultados, poder valorar la intervención del conjunto.

\section{Limpieza}

La limpieza fue quizás el tratamiento más complejo de los llevados a cabo. Como ya se ha apuntado existían diferentes tipos de depósitos y superposiciones por lo que el tratamiento se planteó en distintas fases sucesivas.

Depósitos superficiales no adheridos: En primer lugar el gran acúmulo de polvo se eliminó mediante una limpieza mecánica muy superficial.

Repintes: Durante la limpieza pudo observarse que en numerosos casos debajo de los repintes había pintura original. La mayoría estaban realizados sobre el fijativo 
aplicado en la restauración de 1971-74, de modo que la eliminación de dicho fijativo implicaba su eliminación. El criterio adoptado fue acometer la eliminación de fijativos y repintes conjuntamente y de forma gradual. Finalmente en las zonas donde no había nada debajo se dejaron los repintes, aunque atenuados.

Fijativos: Partíamos de una situación inicial en la que el fijativo, que alteraba enormemente la visión del color, además fijaba en algunas zonas los humos carbonatados, no eliminados en la limpieza de la restauración de los años setenta, y que ocultaban el estrato pictórico. Por otra parte había que tener en cuenta la resistencia del consolidante, su grado de penetración y la extremada fragilidad de la pintura.

Para la limpieza de esta capa pardo-amarilla se hicieron pruebas, basándonos los resultados de los análisis de laboratorio realizadas por el Dr. Parra Crego. El agente disolvente de mayor efecto era el alcohol etílico, aunque la presencia de restos de PVA en la superficie del estrato, procedentes de la consolidación realizada en la restauración anterior, y el estado de la goma laca, muy envejecida e insoluble, hacían muy laboriosa su eliminación. Así pues se aplicó un tratamiento combinado en dos fases consecutivas:

- Carbonato de Amonio aplicado localmente mediante hisopo, ya que ablandaba la capa y mejoraba la efectividad del tratamiento posterior con alcohol. Se descartó la aplicación mediante impacos ya que en tiempos prolongados se enrojecía la película de fijativo ${ }^{16}$ y además, en los grafittis y faltas antiguas, esta coloración afectaba al mortero y resultaba difícil de eliminar. - Alcohol etílico en impaco de pasta de celulosa (Arbocel BC 1000), protegiendo con un film transparente fijado con carboximetil celulosa para evitar la rápida evaporación del disolvente. Retirado el impaco, los restos de goma laca se eliminan con alcohol etílico e hisopo, repasando con bisturí. Los resultados fueron buenos incluso en zonas muy endurecidas aunque los tiempos de permanencia de los impacos variaban mucho según las zonas (Fotografía 4.I).

Humos carbonatados: Una vez eliminada completamente la capa de fijativo y por tanto los repintes que sobre la misma se hicieron y que en este caso eran muy evidentes, se procedió a limpiar el estrato de humo carbonatado (Fotografías 4.I , 4.2, 5.I y 5.2).

Para su limpieza se utilizó EDTA preparado en concentraciones muy bajas y aplicado de forma muy puntual con hisopo para que su permanencia fuese la mínima. Inmediatamente se eliminaba con impacos de agua destilada para frenar la acción del ácido en estratos más profundos.

Aunque el proceso empleado en la limpieza en su conjunto fue muy lento y laborioso los resultados fueron muy buenos y el método de aplicación no resultaba agresivo para la pintura ya que permitía controlar bastante su aplicación, lo que permitió recuperar numerosos detalles de la decoración ocultos por los restos de humo, fijativos y repintes anteriores.

\section{Consolidación}

El proceso de consolidación estuvo condicionado por dos factores fundamentales, por un lado la enorme descohesión del mortero y por otro el problema de oquedades provocado por las filtraciones de las cubiertas. A esto hay que añadir la gran cantidad de lagunas, ya sean consecuencia de pérdidas provocadas por la degradación de los morteros ya sea de forma intencionada o por los abundantes grafittis y agresiones vandálicas.

Antes de iniciar el proceso de consolidación se hizo la fijación y sellado en las zonas de bordes. La protección se hizo con papel japonés y Paraloid y en ocasiones una segunda capa de gasa, proporcionando así una mayor resistencia en la zona a tratar.

Las zonas con decohesión, se trataron con Primal en diferentes porcentajes según su capacidad de penetración. En las oquedades, se utilizó un mortero de inyección, en diferentes porcentajes. Su penetración era aceptable y la consolidación, aun en proporción muy diluida, daba buen resultado.

Se trató que el aporte de humedad fuese mínimo, ya que los tratamientos acuosos provocaban la movilización de las sales internas del muro. La composición de las mismas pudo ser analizada, resultando ser cloruros. Éstas se eliminaron aplicando impacos de celulosa con agua destilada.

\section{Reintegración}

En esta primera fase de la intervención no se quisieron establecer aún los criterios definitivos de reintegración, hasta no comprobar el nivel de pérdidas en una zona más amplia del conjunto. Por ello se adoptaron de forma provisional, atendiendo a su reversibilidad y la posibilidad de intervenir con posterioridad sobre los tratamientos realizados sin perjudicar a la obra.

Los criterios de reintegración material fueron rellenar lagunas a bajo nivel, esta solución también permitiría, llegado el caso, que se pudiera reintegrar con morteros al mismo nivel que la pintura original.

Tanto para el sellado de los bordes de lagunas como para el relleno de lagunas se utilizó un mortero de cal, arena y polvo de mármol.

Con la reintegración cromática, en esta fase, únicamente se pretendía reducir el impacto visual que el excesivo contraste cromático de las lagunas producía en la obra.

En las lagunas reintegradas matéricamente se optó por una única tinta neutra general de un tono muy claro, que matizaba en parte el blanco del mortero y que entonaba con el color que presentaban las zonas de mortero original en las que se había perdido la película pictórica. El tono era muy bajo de forma que podía igualmente servir de base en sucesivas fases del 
trabajo en caso de optar en un futuro por una reintegración más ajustada sea cual fuese la solución adoptada finalmente. El color en estas lagunas se aplicó a base de pigmentos aglutinados con Primal al 5\%.

En las lagunas de película pictórica, tanto las de mayor tamaño, correspondientes a las zonas de infiltraciones, como las menores, a pequeños desgastes, se aplicó una tinta de un color similar al circundante pero de un tono igualmente más bajo. En este caso se emplearon pigmentos aglutinados con goma arábiga preparada al $2 \%$.

A la vista de los resultados en la zona intervenida y de la gran abundancia de lagunas y grafittis que ésta presenta, consideramos que en un futuro sería deseable tender a una reintegración que facilitara la lectura de la obra. Por ello finalmente se ensayaron a posteriori distintas propuestas de reintegración definitiva mediante tratamiento informático de imagen respetando siempre la condición de que la reintegración fuese claramente identificable.

- Aplicando una tinta neutra, aunque de un tono más oscuro que el aplicado en esta primera fase. Aun resultando más neutra la reintegración esta solución tiene el problema de que dada la enorme cantidad de lagunas existentes se pierde la visión de conjunto de la composición, lo cual dificulta su interpretación (Fotografía 6.1).

- Utilizando dos tintas neutras, una más oscura y otra más clara, de forma que el contraste entre las dos serviría para definir los elementos fundamentales de la composición, esta solución sin prestarse en ningún caso a confusión mejora la comprensión de la obra (Fotografía 6.2).

- Reintegración cromática mediante un rigatino ajustando el color en cada una de las lagunas completando la visión de la obra en los casos en que hubiera elementos para su recomposición. Este criterio desde el punto de vista estético permitiría una mejor visión de la obra y por tanto un mejor comprensión de la misma aunque plantea problemas de interpretación debido a la falta de elementos que aportarían los datos necesarios para su reconstrucción (Fotografía 6.3).

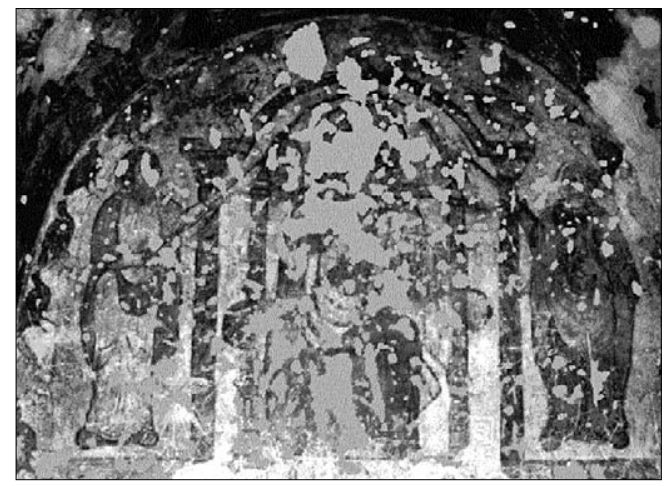

$<$

山
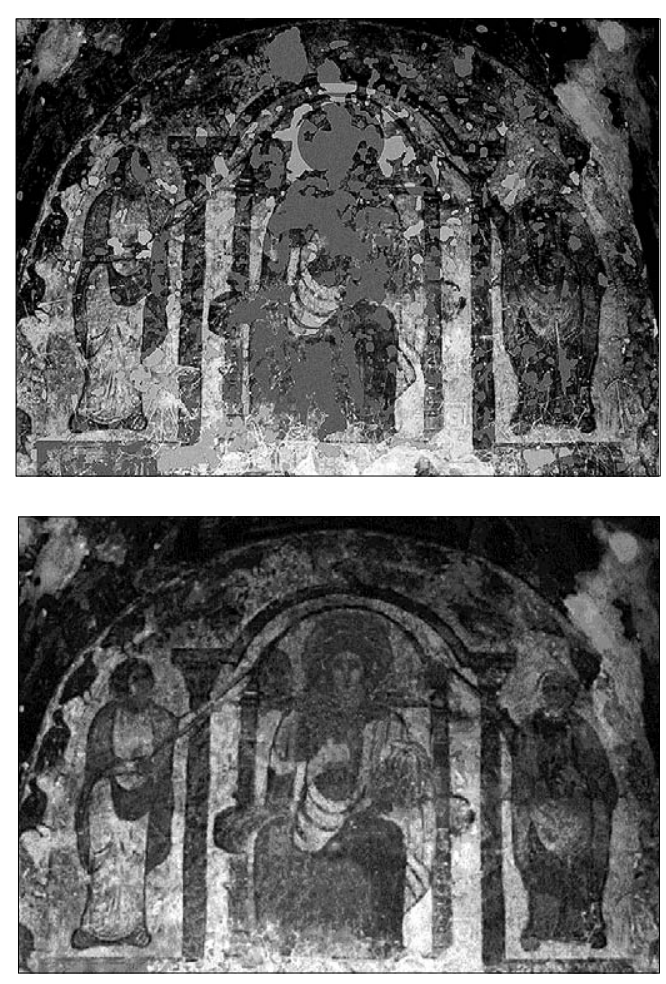

Fotografía 6.3

Fotografía 6.1

Fotografía 6.2

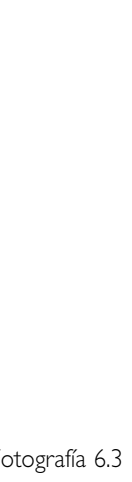

\section{Agradecimientos}

Queremos hacer constar nuestro agradecimiento a todas aquellas personas que han hecho posible de una manera u otra la realización de este trabajo: a D. Antonio Almagro Gorbea Director de la Escuela de Estudios Árabes (CSIC) Granada, a D. Enrique Parra Crego de la Universidad Alfonso X el Sabio, Madrid y al equipo de restauradores participantes en la campaña, D. Francisco José Collado Montero, Dña. Esther Pérez-Aguilera Sánchez, Dña. Isabel Cambil Campaña y Dña. Susana Rodríguez Martín.

\section{Notas}

Véase, ARNOLD, 1965. Painting in Islam. A study of the Place of Pictorial Art in Muslim Culture. CRESWELL, K.A.C. 1979. Compendio de arquitectura paleoislámica, pp. I I 3 - |4I. GRABAR 1981. La formación del Arte islámico. GRABAR 1964. Islamic Art and Byzantium. Dumbarton Oaks papers 18, pp. 69 y ss.
2. ALMAGRO, M.; CABALLERO, L; ZOZAYA, J.; y ALMAGRO, A. 1975. Qusayr 'Amra. Residencia y baños omeyas en el desierto de Jordania. Ministerio de Asuntos Exteriores. p. 57.

3. Ibidem p. 57 
4. BLÁZQUEZ, J.M. 198I. Las pinturas helenísticas de Qusayr 'Amra, (Jordania) y sus fuentes. Archivo Español de Arquelolgía. pp. $160-163$.

5. ALMAGRO. pp. 163-179.

6. BLÁZQUEZ, J.M. 1981. Op. Cit. Archivo Español .... № |43144. pp. 158.

7. MORA, P.; MORA, L. y PHILLIPOT, P. 1984; Conservation Wall Paintings, pp. 108-1 10

8. Se sabe que las pinturas murales de Qusayr 'Amra fueron intervenidas con anterioridad en varias ocasiones. El texto de Martín Almagro y otros, Qusayr 'Amra. Residencia y Baños Omeyas en el desierto de Jordania, refiere una intervención que se desarrolló durante los 197|-74 "Este equipo trabajó durante una primera campaña de tres meses, procurando análisis de colores, datos sobre la técnica pictórica utilizada y logró encontrar el mejor procedimiento para limpiar y fijar las pinturas. Este trabajo se prosiguió en dos campañas sucesivas hasta dejar absolutamente limpia y consolidada la totalidad de la ornamentación pictórica mural del monumento. Se cuidó en esta primera etapa de respetar todo lo conservado y dejar visible también las partes perdidas de las ricas pinturas que ornaron las paredes del monumento. Solo se dio una tinta neutra en aquellas superficies en que se había perdido totalmente la decoración en espera de decidir acerca de una posible reintegración, al menos parcial, de esas zonas" (p. I I2)

Igualmente, se realizó una segunda intervención, aunque en este caso se trató fundamentalmente de eliminar los testigos de suciedad que se habían dejado en la anterior restauración así como realizar algún tratamiento local.

No se conocen más intervenciones, pero es muy probable que haya habido alguna otra más, aunque no coordinada por la Misión Española.
9. ABAD CASAL, L. 1982. Lucentum I Aspectos técnicos de la pintura mural romana, pp. I52-I54. Anales de la Universidad de Alicante, pp. 143 y 144.

10. MORA, P.; MORA, L.; PHILLIPOT, P. 1984. Conservation of Wall Paintings. Butterworhs. pp. 73-75.

I I. GÁRATE ROJAS, I. 1994. Las artes de la cal. Ministerio de Cultura, I.C.R.C.B. pp. 122-125.

12. La determinación de materiales orgánicos ha sido realizada por el Dr. Parra Crego, de la Universidad Alfonso X el Sabio de Madrid.

13. En la documentación referida (Martín Almagro y otros, p. 7I) se apunta que la técnica de ejecución era al seco, sobre una preparación similar a la del fresco.

14. Véase el estudio de aglutinantes empleados en las pinturas murales de la Tumba de Nefertari realizado por Antoni Palet (VIII Congreso de Conservación de Bienes Culturales, 1990), en el que también se identifica como aglutinante una variante de goma arábiga local por la falta de un polisacárido, la Ramnosa, demostrando su obtención de las acacias nilóticas de Qrna.

15. La existencia de zonas arrancadas se documenta en la obra ya citada de Martín Almagro et al. Se conoce la existencia de un fragmento arrancado de estas pinturas en el museo de Berlin.

16. Algunos componentes de la goma laca, sobre todo cuando esta no está bien purificada, enrojecen en medio básico, probablemente es lo que ocurre en este caso. 